\title{
Wearable Devices and Biosensing: Future Frontiers
}

\author{
Peter R. Chai ${ }^{1}$
}

Received: 13 June 2016 / Accepted: 17 June 2016 / Published online: 28 June 2016

(C) American College of Medical Toxicology 2016

Keywords Biosensors · Telemedicine $\cdot$ Wearable devices · Technology $\cdot$ Body sensors

Since the 1950s, toxicologists have utilized novel technologies to advance our understanding of poisonings while improving our availability to physicians and patients [1]. From rolodexes and telephones to head mounted computers, advanced biosensing, and ingestible sensors, toxicologists have always been pioneers leveraging advanced technologies to solve problems [1, 2]. As smartphones, fitness monitors, and connected devices become ubiquitous, toxicologists are naturally equipped with advanced tools that augment our bedside exam of poisoned patients.

A new generation of toxicologists continually pushes the boundaries of technology in an effort to facilitate improved patient care and access to our expertise [1-5]. Head-mounted wearable computers can provide a toxicologist with a firstperson view of a poisoned patient, while a wrist-mounted sensor can stream key biometric data (e.g., heart rate, respiratory rate, skin temperature, and electrodermal activity). Ingestible biosensors can provide historical records of medication ingestion, and linked webcams can stream toxicology lectures to centers seeking expertise on the poisoned patient $[2-4,6]$.

In an era of integrated care and bundled payments, toxicologists and fellows in training have a unique opportunity to develop novel technology-based methods that respond to a

Peter R. Chai

peter.chai@umassmemorial.org

1 Division of Medical Toxicology, Department of Emergency Medicine, University of Massachusetts Medical School, 55 Lake Ave North, Worcester, MA 01655, USA need in our specialty. Creating novel applications using everyday technology requires a contemporary approach - integration of patients, physicians, engineers, and software developers into a multidisciplinary research team.

\section{Integrating Biosensor Data with Patients}

Wearable biosensors, like Fitbits, noninvasively collect realtime biometric data. Each wearable device or biosensor provides an additional stream of data on our patients. As wearable biosensors become accepted and commonplace in patients $95 \%$ of emergency department patients interact daily with smartphones - they can be leveraged to gather important data correlated with various disease processes [7]. Integration of a suite of devices can provide a comprehensive profile of the poisoned patient which, when evaluated remotely, provides increased precision and effectiveness of toxicologists [8]. Changes in heart rate using a wearable biosensor in a patient at a critical access hospital may signal worsening calcium channel blocker toxicity; a wearable camera may help a toxicologist guide the bedside clinician in the administration of physostigmine for the anticholinergic patient, and the virtual assessment of an altered patient can change a toxicologist's recommendation to triage a patient to an inpatient bed or emergency department observation period [3].

Much like endocrinologists use insulin pump data in diabetics, toxicologists who practice substance abuse treatment can use biosensor data to discover episodes of relapse and tolerance $[4,9]$. Advanced algorithms and improving technology can provide a noninvasive, yet accurate understanding of real-time substance abuse and relapse. This knowledge may help toxicologists tailor substance abuse counseling, by understanding real-time triggers of tolerance, addiction, sobriety, and relapse [9]. 
Integrating advanced technology tools in patient care will require nontraditional evaluation methods including patientcentered acceptability, hardware design, and engineering [10]. The accuracy of biosensor data is dependent on how patients interact with these devices and sampling accuracy of the device, for example, the promise of remote heart rate tracking with commercial wearable biosensors has revealed variable diagnostic accuracy [11-13].

\section{Opportunities for Toxicologists}

Toxicologists play important roles in the evaluation of advanced technology. As bedside diagnosticians who care for a wide variety of patients in person and remotely, our experience evaluating biosensor data can improve our ability to care for our patients, anticipate adverse events, and monitor medication safety $[3,4,6,9]$. Capitalizing on these experiences provides a good opportunity for research evaluating and helping to develop new devices.

New research opportunities bring similar challenges as traditional research and toxicologists who initiate investigations in biosensor research will require funding. A variety of funding mechanisms through the American College of Medical Toxicology (ACMT), Medical Toxicology Foundation (MTF), and American Academy of Clinical Toxicology (AACT) in addition to traditional NIH funding mechanisms exist to support junior investigators in nascent investigations. Technology-based investigations are especially relevant in toxicology and substance abuse and are recently identified priorities by the National Institute on Drug Abuse (NIDA) in an effort to understand and intervene in the opioid epidemic and HIV-related substance abuse [14, 15]. Gaining expertise in technology development and evaluation can be of particular appeal to fellows in training by providing a skill that is easily translated into various fields of medicine.

\section{What's Next in Technology Studies for Toxicologists?}

As evaluation of advanced technology wearable devices yield discrete data streams, the toxicologist will need to decide how to integrate disparate data streams in the context of the patient. Virtual bedside exams combined with multiple streams of biosensor data can provide a toxicologist with a comprehensive overview of a patient. Integrated technology begins to allow toxicologists to anticipate the course of a poisoned patient, deliver tailored therapy, and offer guidance to bedside clinicians. In substance abuse, the integration of multiple biosensor streams give a toxicologist new tools to evaluate tolerance, dependence, and addiction. As technology continues to intercalate itself into our daily lives, we should leverage an advancing field of wearable devices and biosensors to improve the way in which we care for our patients.

Acknowledgments Special thanks to Dr. Christine Murphy for her assistance in editing this manuscript.

\section{Compliance with Ethical Standards}

Conflict of Interest The authors declare that they have no competing interests.

Sources of Funding The author is supported by the Medical Toxicology Foundation.

\section{References}

1. Skolnik A. Telemedicine and toxicology: back to the future? J Med Toxicol. 2013;9:217-9.

2. Wong A, Vohra R, Ruha A-M, Koutsogiannis Z, Graeme K, Dargan PI, et al. The Global Educational Toxicology Uniting Project (GETUP): an analysis of the first year of a novel toxicology education project. J Med Toxicol. 2015;11:295-300.

3. Chai PR, Babu KM, Boyer EW. The feasibility and acceptability of Google glass for teletoxicology consults. J. Med. Toxicol. 2015 ed. Springer US; 2015;11:283-7.

4. Carreiro S, Smelson D, Ranney M, Horvath KJ, Picard RW, Boudreaux ED, et al. Real-time mobile detection of drug use with wearable biosensors: a pilot study. J Med Toxicol. 2014;11:73-9.

5. Maddry JK, Sessions D, Heard K, Lappan C, McManus J, Bebarta VS. Wartime toxicology: evaluation of a military medical toxicology telemedicine consults service to assist physicians serving overseas and in combat (2005-2012). J Med Toxicol. 2014;10:261-5.

6. Chai PR, Castillo-Mancilla J, Buffkin E, Darling C, Rosen RK, Horvath KJ, et al. Utilizing an ingestible biosensor to assess realtime medication adherence. J. Med. Toxicol. 2015 ed. Springer US; 2015;1-6.

7. Ranney ML, Choo EK, Wang Y, Baum A, Clark MA, Mello MJ. Emergency department patients' preferences for technology-based behavioral interventions. Ann Emerg Med. 2012 ed. 2012;60:21848.

8. Chai PR, Wu RY, Ranney ML, Porter PS, Babu KM, Boyer EW. The virtual toxicology service: wearable head-mounted devices for medical toxicology. J. Med. Toxicol. 2014 ed. 2014;10:382-7.

9. Carreiro S, Fang H, Zhang J, Wittbold K, Weng S, Mullins R, et al. iMStrong: deployment of a biosensor system to detect cocaine use. J Med Syst. 2015;39:186.

10. Birnbaum F, Lewis D, Rosen RK, Ranney ML. Patient engagement and the design of digital health. Miner J, editor. Acad Emerg Med. 2015;22:754-6.

11. Nelson MB, Kaminsky LA, Dickin DC, Montoye AHK. Validity of consumer-based physical activity monitors for specific activity types. Med Sci Sports Exerc. 2016;1.

12. Singh AK, Farmer C, Van Den Berg MLE, Killington M, Barr CJ. Accuracy of the FitBit at walking speeds and cadences relevant to clinical rehabilitation populations. Disabil Health J. 2016;9:320-3.

13. Crouter SE, Albright C, Bassett DR. Accuracy of polar S410 heart rate monitor to estimate energy cost of exercise. Med Sci Sports Exerc. 2004;36:1433-9. 
14. National Institutes of Health, Research OOA. NIH HIV/AIDS research priorities and guidelines for determining AIDS funding [Internet]. 2015 [cited 2016 Feb 26]. Available from: http://grants. nih.gov/grants/guide/notice-files/NOT-OD-15-137.html
15. Compton WM, Volkow ND, Throckmorton DC, Lurie P. Expanded access to opioid overdose intervention: research, practice, and policy needs. Ann Intern Med. 2013;158:65-6. 